\title{
A rare case of double infarction treated with primary percutaneous coronary intervention
}

\author{
Biplab Paul, Pranab K. Biswas, Biswajit Majumder, Debojyoti Sarkar, Aritra Konar \\ R. G. Kar Medical College, Kolkata, India
}

Postep Kardiol Inter 2015; 11, 3 (41): 230-232

DOI: $10.5114 /$ pwki.2015.54021

\section{Introduction}

Coronary artery disease is the leading cause of mortality and morbidity in developing as well as developed countries, and the acute presentation occurs in the form of ST-elevation myocardial infarction (STEMI) or unstable angina (UA)/non-ST-elevation myocardial infarction (NSTEMI). The process of acute myocardial infarction begins with an anatomic or functional obstruction in the coronary vascular bed, which results in regional myocardial ischemia and, if the ischemia persists, in infarction. Depending on the involved vessels, e.g., left anterior descending (LAD), left circumflex (LCX) or right coronary artery (RCA), it results in anterior wall myocardial infarction (AWMI), inferior wall myocardial infarction (IWMI), or right ventricular myocardial infarction (RVMI), etc.

Acute myocardial infarction (AMI) involving two or more culprit lesions at the same time, known as double or combined infarction, is an extremely rare event with a grave prognosis [1, 2]. Here, we describe a case of a patient with acute myocardial infarction due to total occlusion of the left anterior descending and right coronary artery who presented with cardiogenic shock.

\section{Case report}

A 34-year-old young male smoker presented with sudden onset severe retro-sternal chest pain lasting for $2 \mathrm{~h}$ with radiation to the left $\mathrm{arm}$. On examination the pulse was 110/min and blood pressure was $70 \mathrm{~mm} \mathrm{Hg}$ (systolic); bilateral basal crackles were present along with left ventricular third heart sound. ECG showed ST elevation in anterior leads (V1-V6) and also in inferior leads (II, III, aVF) (Figure 1), and troponin T was elevated. The patient also had several episodes of ventricular tachycardia alternating with complete heart block. Based on history and clinical examination, we made a diagnosis of acute anterior wall myocardial infarction, probably due to occlusion of the left anterior descending coronary artery, which had also wrapped around the inferior wall. The patient was loaded with $350 \mathrm{mg}$ of aspirin and $600 \mathrm{mg}$ of clopidogrel and was immediately shifted to the cath lab for coronary angiography with the intention to do primary percutaneous coronary intervention ( $\mathrm{PCl}$ ). A temporary pacemaker lead was inserted as he developed persistent complete heart block.

But, after performing coronary angiography, we were surprised to find that both the LAD (Figure $2 \mathrm{~A}$ ) and the RCA (Figure 3 A) were totally occluded, which seemed to be acute as there was absence of collateral and presence of thrombus. The patient was loaded with 5000 IU of unfractionated heparin (UFH) (body weight $70 \mathrm{~kg}$ at a dose of approx $70 \mathrm{U} / \mathrm{kg}$ ); the left coronary artery was engaged with an EBU 3.5 $7 \mathrm{Fr}$ catheter and a stabilizer soft guidewire was passed. An intravenous bolus of eptifibatide was given at $180 \mu \mathrm{g} / \mathrm{kg}$ was given and infusion was started at $2 \mu \mathrm{g} / \mathrm{kg} / \mathrm{min}$. Thrombosuction was done with a $6 \mathrm{Fr}$ Export AP ASPIRATION Catheter (Medtronic) and then the lesion of the proximal LAD was predilated with a $2 \times$ $10 \mathrm{~mm}$ Sprinter Legend balloon. A $3 \times 24 \mathrm{~mm}$ Endeavor Sprint stent was deployed at 16 atm. By that time, the patient developed two episodes of sustained ventricular tachycardia and was reverted with $100 \mathrm{~J}$ of DC shock.

Then the right coronary artery was engaged with a JR 3.5 $7 \mathrm{Fr}$ catheter and a stabilizer soft guidewire was introduced. Thrombosuction was done with an Export AP ASPIRATION catheter and a $3.5 \times 30 \mathrm{~mm}$ Endeavor Sprint stent was deployed at $14 \mathrm{~atm}$. There was no dissection, no residual stenosis, and TIMI 3 flow was established in both the LAD (Figure 2 B) and RCA (Figure 3 B). Potential sources of hypercoagulability were investigated, including lupus anticoagulant, anticardiolipin antibodies, factor $\mathrm{V}$ Leiden, myeloproliferative disease and cancer, all of which were negative. Serum homocysteine level was normal and lipid profile was mildly deranged.

\section{Corresponding author:}

Biplab Paul MD, R. G. Kar Medical College, Room No-48, Kb Hostel, R. G. Kar Medical College, 700004 Kolkata, India, fax: 919674941651 , e-mail: biplov_107@yahoo.in

Received: 6.06 .2014 , accepted: 30.09 .2014$. 

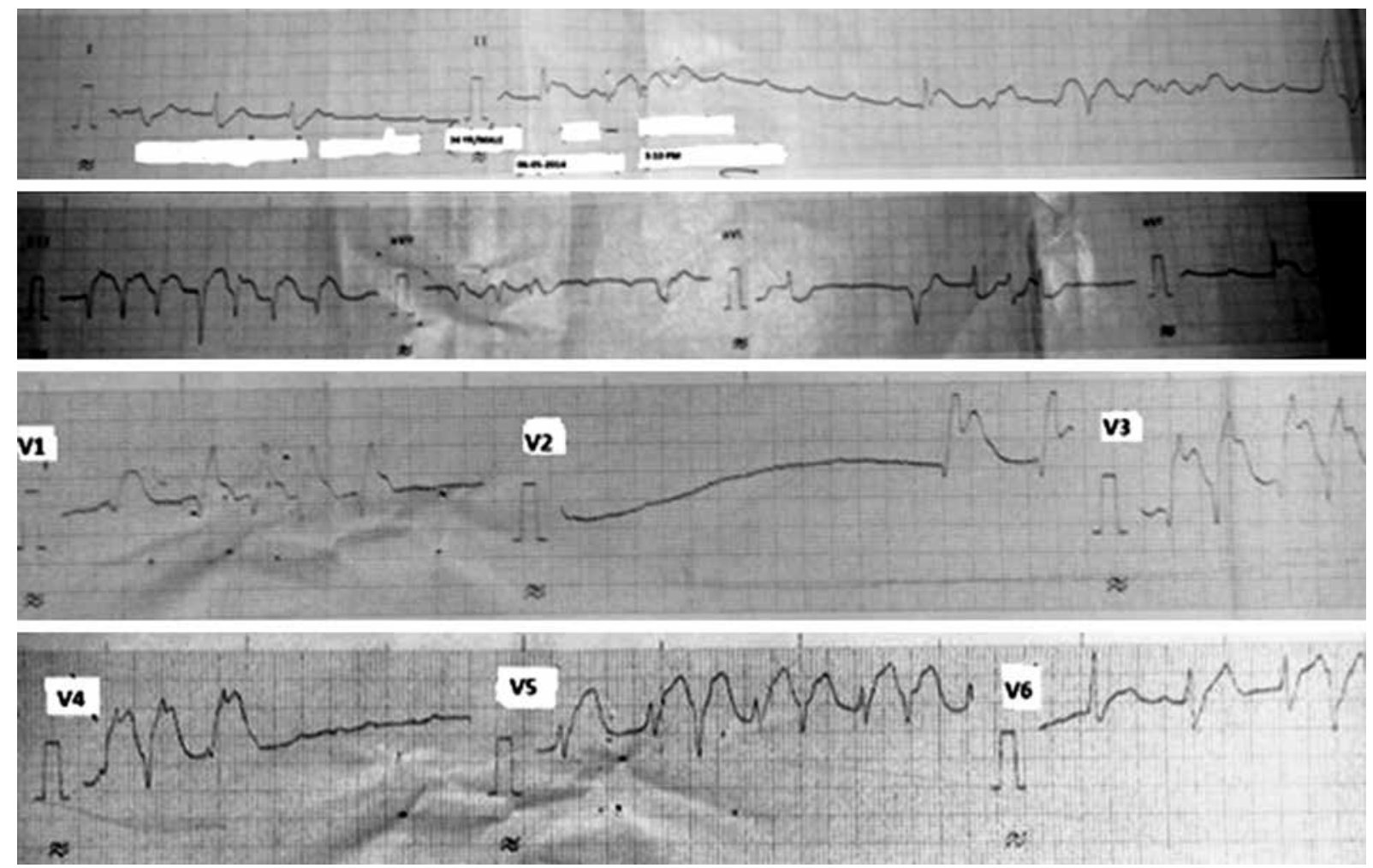

Figure 1. ECG showing ST elevation in lead II, III, AvF and V1-V6 and intermittent complete heart block
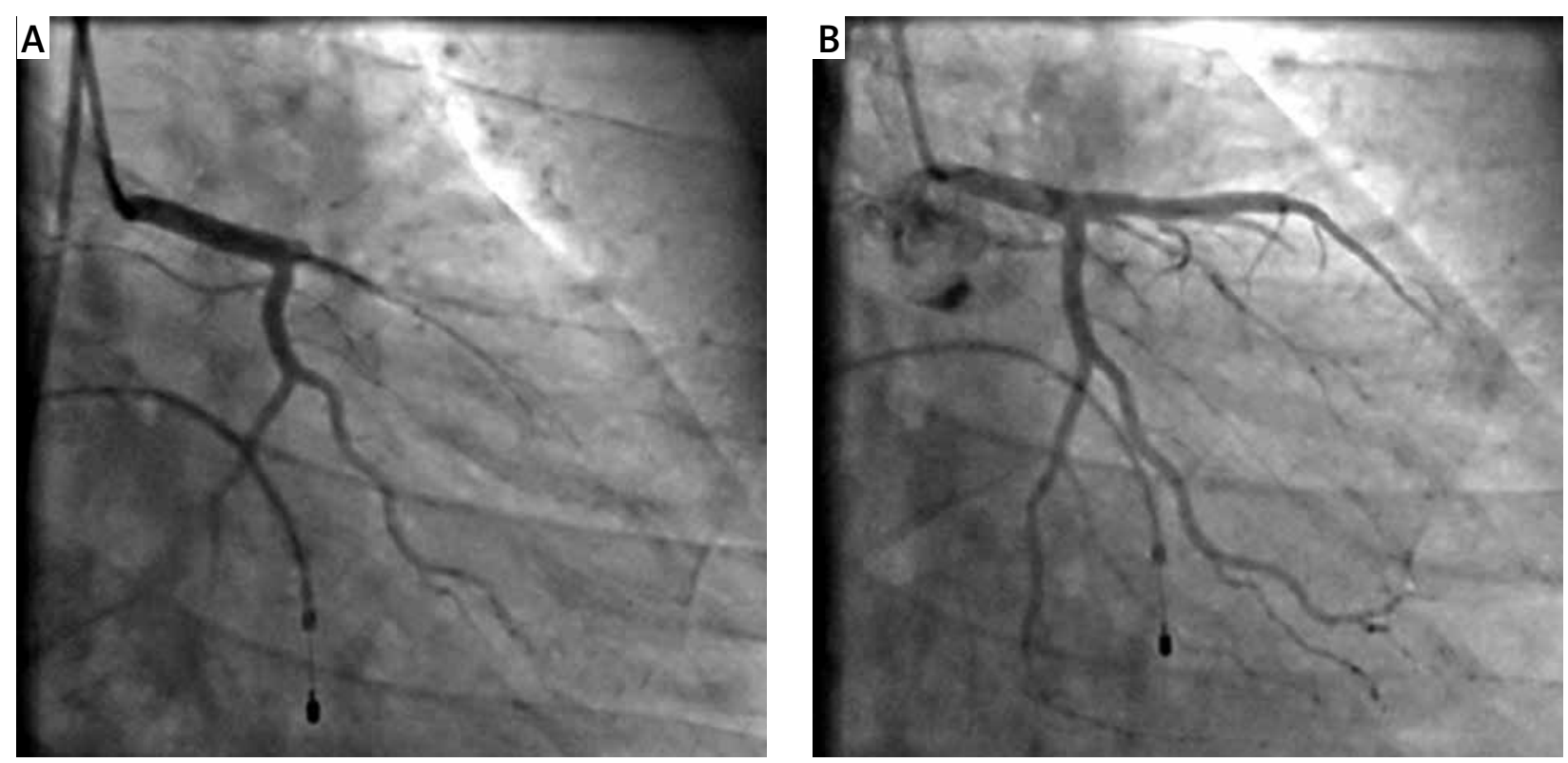

Figure 2. RAO caudal view showing total occlusion of LAD coronary artery (A), which was opened later (B)

\section{Discussion}

Acute myocardial infarction as a consequence of acute transmural ischemia of two vascular territories at the same time is known as double or combined infarction $[1,2]$. Although, sometimes, we may find ECG evidence of both anterior and inferior myocardial infarction due to occlusion of "wraparound" LAD, double infarction due to occlusion of two separate vessels is extremely rare. In the literature, the actual incidence is not known and studies are limited. Pollak et al. [6] found 18 cases (2.5\%) of multiple culprit arteries in a series of 711 patients undergoing primary $\mathrm{PCl}$. Such a low incidence may be related to the fact that AMI with multiple vessel obstruction often causes extensive myocardial injury and death occurs before the patient arrives at the hospital. Again, in those 

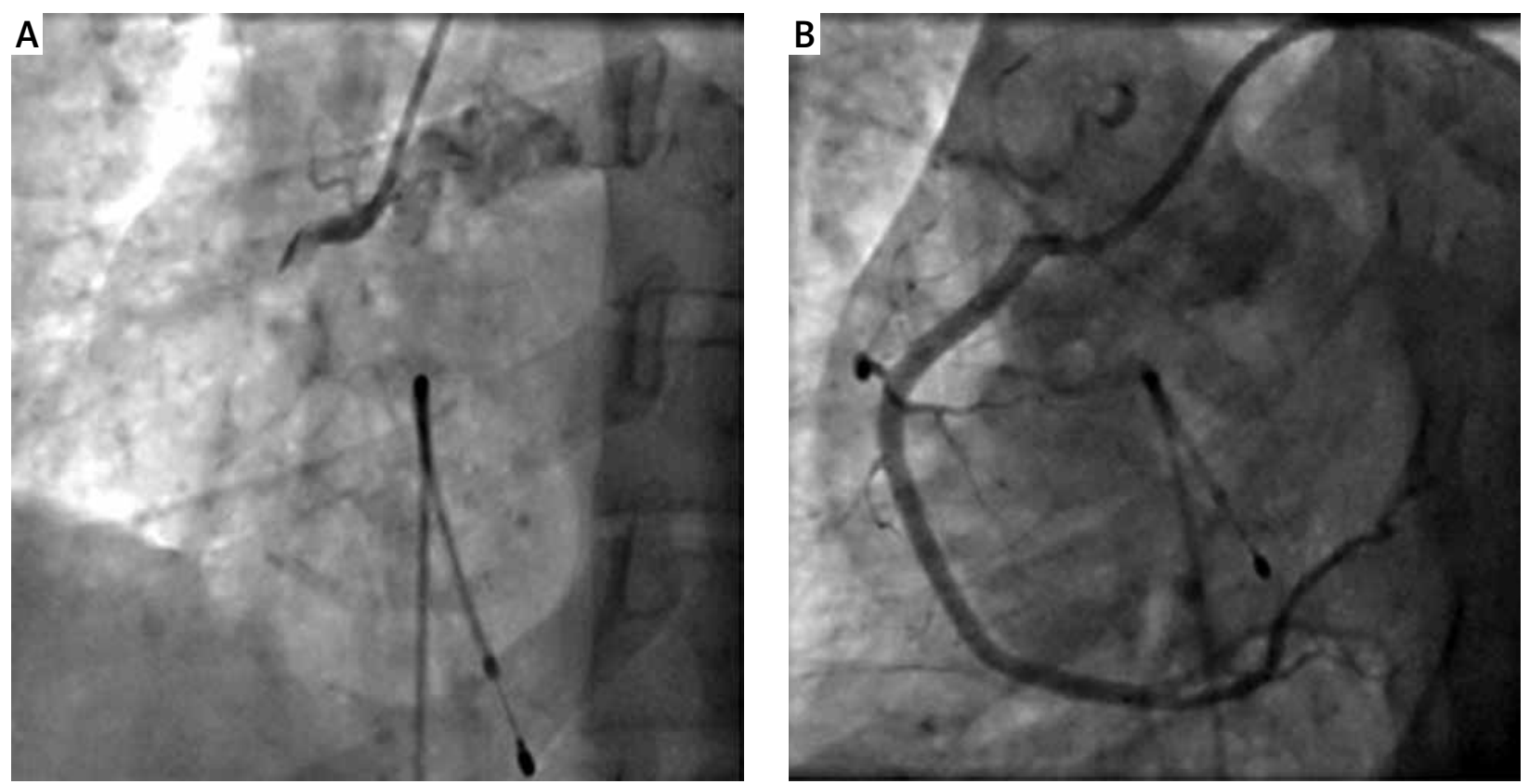

Figure 3. LAO view showing total occlusion of RCA along with thrombus and meniscus sign (A), which was opened later (B)

patients who undergo thrombolysis and do not undergo coronary angiography, culprit vessel information of these patients is also not known. It is speculated that double infarction results from the overall prothrombotic and inflammatory conditions associated with AMI.

Now the important question is: what are the factors responsible for simultaneous acute thrombosis of multiple coronary arteries? Although these are not fully known, possible explanations include: (1) heightened inflammatory response and catecholamine surge caused by acute occlusion of one vessel, leading to occlusion of another vessel; (2) hemodynamic instability and hypotension due to occlusion of one coronary artery, resulting in blood stasis and acute occlusion of another artery with a severe underlying lesion (commonly LAD followed by RCA occlusion); (4) prolonged coronary vasospasm (due to Prinzmetal angina or cocaine use); (3) hypercoagulable states including malignancy, hyperhomocysteinemia and thrombocytosis; and (4) coronary embolism and coronary arteritis [3-6].

Regarding management of these patients, whether thrombolysis or primary $\mathrm{PCl}$ is preferred is not known. However, as it is probably due to a heightened thrombotic state, primary $\mathrm{PCl}$ may be a better choice than thrombolysis. In spite of successful thrombolysis or $\mathrm{PCl}$, almost one third of patients die, and therefore they should be aggressively treated and extremely good supportive care is also needed to save valuable lives.

\section{Acknowledgments}

I am grateful to all the doctors of the Department of Cardiology of R. G. Medical College for their help and support.

\section{Conflict of interest}

The authors declare no conflict of interest.

\section{References}

1. Ribeiro H, Ferreira C, Batista A, et al. Sequential double vessel myocardial infarction. Rev Port Cardiol 2011; 30: 925-7.

2. Sia SK, Huang CN, Ueng KC, et al. Double vessel acute myocardial infarction showing simultaneous total occlusion of left anterior descending artery and right coronary artery. Circ J 2008; 72: 1034-6.

3. Shen AY, Mansukhani PW, Aharonian V. Primary angioplasty for acute myocardial infarction resulting from the simultaneous occlusion of two major coronary arteries. Catheter Cardiovasc Interv 1999; 47: 203-7.

4. Yoshitomi Y, Kojima S, Kuramochi M. Acute myocardial infarction with simultaneous occlusions of two major coronary arteries in a young man. Clin Cardiol 1998; 21: 140-2.

5. Davies MJ, Thomas A. Thrombosis and acute coronary-artery lesions in sudden cardiac ischemic death. N Engl J Med 1984; 310: $1137-40$

6. Pollak PM, Parikh SV, Kizilgul M, Keeley EC. Multiple culprit arteries in patients with ST segment elevation myocardial infarction referred for primary percutaneous coronary intervention. Am J Cardiol 2009; 104: 619-23. 